\title{
$\frac{\mathrm{DE}}{\mathrm{G}}$
}

\section{Evaluating Injury Risk in First and Second League Professional Portuguese Soccer: Muscular Strength and Asymmetry}

\author{
by \\ Alberto Carvalho', Scott Brown ${ }^{2}$, Eduardo Abade ${ }^{1}$
}

Strength imbalances between the hamstrings and quadriceps are an essential predictor for hamstring strain in soccer. The study aimed to investigate and compare the muscle strength imbalances of professional soccer players of different performance levels. One hundred and fifty nine senior male professional soccer players from first $(n=75)$ and second league $(n=84)$ Portuguese clubs participated in this study. Muscle strength was evaluated with a REV9000 isokinetic dynamometer. Maximal peak torque data were used to calculate quadriceps and hamstrings strength during concentric and eccentric actions, bilateral asymmetry, conventional strength ratios and dynamic control ratios. Second league athletes produced slightly lower conventional strength ratios in the right and left legs ( $E S=0.22, p=0.17$ and $E S=0.36, p=0.023$, respectively) compared to the first league athletes. No significant differences were found in dynamic control ratios or in bilateral asymmetry among first and second league athletes. These findings do not show a clear link between the competitive level and injury risk in soccer players. However, some of the differences found, particularly in conventional strength ratios, highlight the importance of performing off-season and pre-season strength assessments to prescribe and adjust individual strength training programs among professional soccer players.

Key words: dynamometry; isokinetic strength; H:Q ratios; muscle imbalances.

\section{Introduction}

Muscular injuries to the lower extremities, particularly hamstrings strains, are common in soccer (Ekstrand et al., 2011). A high number of intense high-speed actions such as accelerating, decelerating, jumping, turning and kicking (Bangsbo et al., 2006) require the hamstrings to perform in a position of extreme stretch. This means that significant muscle strength imbalances may increase muscular discomfort and result in high injury risk. Therefore, adequate training and prevention methods are essential to predict injury risk and decrease the probability of an athlete being unable to participate in training or match play. Thus, it is important to understand if the match and training level (i.e. professional, semi- professional or amateur) may affect the injury risk in soccer players.

Dynamometers are commonly used to clinically assess the physiological function of muscular strength and are widely regarded as the "gold standard" in the examination of concentric (con) and eccentric (ecc) actions, providing researchers with a valid (Drouin et al., 2004) assessment tool across a wide-range of parameters (i.e. joint, velocity, range of motion and action). Over the last three decades, researchers have confidently affirmed the reliability of many different dynamometers such as Biodex (Ordway et al., 2006), Cybex (Impellizzeri et al., 2008), Kin Com (Sole et al., 2007), Con-Trex (Bardis et al.,

\footnotetext{
1 - Research Center in Sports Sciences, Health Sciences and Human Development, CIDESD, University Institute of Maia, ISMAI, Portugal.

2 - Sports Performance Research Institute New Zealand (SPRINZ), Auckland University of Technology, Auckland, New Zealand.
} 
2004), Merac (Capranica et al., 1998), Lido (Iga et al., 2006), iSAM 9000 (Orri and Darden, 2008) and REV9000 from Technogym (Carvalho et al., 2013). Using these devices, evaluations of strength during isometric and isokinetic actions have been performed (Bardis et al., 2004) at the knee to assess the risk of injury and to develop rehabilitation programmes for knee and hamstring injuries and/or strength imbalances (Croisier, 2004; Impellizzeri et al., 2008).

Muscle strains, particularly in the hamstrings, are prevalent in sports like soccer that involve intense high-speed movements (e.g. sprinting) and generally occur during the terminal swing-phase of the gait cycle (Orchard, 2002). Sport-specific actions, such as kicking, require the hamstrings to perform in a position of extreme stretch (Askling et al., 2007). Among the hamstrings, the biceps femoris is known to experience the greatest musculotendon stretch (Thelen et al., 2005), suggesting a greater risk of injury compared to the semimembranosus and semitendinosus muscles during high-speed running. The critical moment of injury occurs during the second half of a swing when the hamstrings lengthen and absorb the energy of the decelerating leg (Chumanov et al., 2011).

It is well accepted that a strength imbalance between the hamstrings and quadriceps is an essential predictor for hamstring strain (Croisier et al., 2002) and anterior cruciate ligament injuries (More et al., 1993). A hamstrings to quadriceps strength ratio based on concentric peak torque values (H:Q) has been explored (Brown et al., 2014) to describe knee-joint stability. In fact, it has been suggested (Heiser et al., 1984) that injury prevention strategies, based on the detection of H:Q imbalances, should aim for a normative value of 0.60 at an angular velocity of $60^{\circ} \cdot \mathrm{s}-1$. However, this conventional strength ratio (CSR) seems to be insufficient and inadequate in describing functional movements involving knee extension and flexion. As such, a dynamic control ratio (DCR) has been established to incorporate a more functional evaluation of unilateral or withinleg strength imbalances. It is thought that the strength relationship between knee extension and flexion is better described by a DCR of eccentric hamstrings to quadriceps concentric muscle strength (e.g. knee extension; Hecc:Qcon) or concentric hamstrings to eccentric quadriceps muscle strength (e.g. knee flexion; Hcon:Qecc) (Aagaard et al., 1995). A DCR of 1.00 has been proposed (Coombs and Garbutt, 2002) as a predictor to indicate a significant capacity of the hamstrings to provide joint stabilization during knee extension.

In addition to unilateral strength imbalances, bilateral or between-leg asymmetry in lower extremity strength has been stated (Burkett, 1970) as the source of several injuries, especially hamstring strains. Bilateral asymmetry under $5 \%$ suggests good muscular status (Croisier and Crielaard, 2000), which may reduce muscular discomfort and subsequent hamstring injury (Croisier et al., 2008). Thus, this assessment of strength differences in the lower extremities is an important screening tool for injury risk and a valuable reference in the prescription of rehabilitation programs (Cheung et al., 2012).

During the competitive season, the number of matches performed by second league athletes is higher when compared to the first league (42 games vs 30 games, respectively). However, to our knowledge, athletes from first and second Portuguese leagues experience similar training methodologies that include specific strength training for injury prevention. Despite this information about training and match play profiles, the relationship between the competitive level and the injury risk is not fully understood. Particularly, the hypothetical influence of different enrolled demands on muscle imbalances and risk of injury is unknown. Based on these assumptions, this study aimed to investigate and compare the muscle strength imbalances in professional soccer players of different performance levels.

\section{Material and Methods}

\section{Participants}

One hundred and fifty nine senior male professional soccer players from first $(n=75$, age $25 \pm 5$ yrs, body height $1.8 \pm 0.1 \mathrm{~m}$, body mass $74 \pm$ $7 \mathrm{~kg})$ and second league $(\mathrm{n}=84$, age $26 \pm 5 \mathrm{yrs}$, body height $1.8 \pm 0.1 \mathrm{~m}$, body mass $76 \pm 7 \mathrm{~kg}$ ) Portuguese clubs volunteered as participants for this study and consisted of side backs, central backs, central defending midfielders, central attacking midfielders and forwards. Goalkeepers were not included in this study as they had recently been found to possess unique strength 
profiles that are unrepresentative of all other field positions (Ruas et al., 2015). Athletes with current lower extremity injury or who were currently performing rehabilitation processes were excluded from the analysis. Prior to testing, all athletes read and signed informed consent documents as approved by the institutional review board (ethics committee of the Research Center in Sports Sciences, Health and Human Development, Vila Real, Portugal).

\section{Measures}

Carvalho et al. (2013) showed high reproducibility (i.e. an intraclass correlation coefficient) of eccentric (ICC, 0.95-0.97), concentric (ICC, 0.95-0.96) and isometric (ICC, 0.93-0.96) strength for knee extensor and flexor muscles when using REV9000. Thus, the REV9000 isokinetic device provides high reproducibility to evaluate isometric and dynamic strength of the knee extensors and flexors.

\section{Procedures}

The experimental protocol was performed in July during the second week of the preseason. All testing sessions took place at the same time of the day (from 9:30 to 11:30 am) following a minimum of 24 hours without training and lasted approximately $10 \mathrm{~min}$ per athlete.

All athletes performed a 10 min warm-up on an ergocycle (70 rpm at $50 \mathrm{~W}$ ) and dynamic stretching exercises (for hamstrings and quadriceps) followed by adequate familiarization with the dynamometer with five isokinetic flexion/extension repetitions at $90^{\circ} \cdot \mathrm{s}-1$. The isokinetic assessment was performed using a REV9000 (Technogym SpA, Cresena ITA) isokinetic dynamometer where the athletes were placed in a seated position with a trunk angle of $85^{\circ}$ and the trunk, thigh and distal shank were stabilized with belts. The mechanical axis of the dynamometer was aligned with the lateral epicondyle of the knee and the lever arm was positioned at the distal third of the leg. A total knee range of motion of $70^{\circ}$ was determined with full anatomical knee extension as $0^{\circ}$ (i.e. $20^{\circ}$ of extension to $90^{\circ}$ of flexion). The testing protocol consisted of five concentric flexion/extension actions at $60^{\circ} \cdot \mathrm{s}-1,2 \mathrm{~min}$ rest and five eccentric extension/flexion actions at $60^{\circ} \cdot \mathrm{s}-1$ of the right and left legs (Brown et al., 2014). The gravitational factor of the dynamometer's lever arm and lower extremity ensemble were calculated by the dynamometer and automatically compensated for during the measurements. Verbal encouragement was given, but the athlete did not receive any visual feedback during the test.

Maximal peak torque data were used to calculate quadriceps and hamstring strength during concentric and eccentric actions, bilateral asymmetry, the CSR and DCR. Unilateral CSRs were calculated by the ratio between the peak concentric torque of the hamstrings divided by the peak concentric torque of quadriceps. Unilateral DCRs were calculated by the ratio between the peak eccentric torque of the hamstrings divided by the peak concentric torque of the quadriceps (Impellizzeri et al., 2008). Previous investigators (Kuruganti and Seaman, 2006) had presented several different formulas to calculate the bilateral lower extremity strength asymmetry. In the present study, absolute bilateral muscle strength differences were calculated according to the formula: ((peak torque of the stronger leg - peak torque of the weaker leg) / peak torque of the weaker leg) ${ }^{*} 100$ ).

\section{Statistical Analysis}

Athletes' characteristics, peak torque, asymmetry, CSR and DCR data is presented as means \pm SD. The data were plotted through a boxplot to identify any outliers and their normality was assessed via a Shapiro-Wilk test. Once the data were determined as normally distributed, a 2-tailed heteroscedastic Student's ttest was used to compare means. The probability of type I error (alpha) was set at priori at 0.05 in all statistical analysis. The procedures were performed with SPSS statistical software (Version 19.0 for Windows, SPSS Inc., Chicago, IL, USA). Additionally, effect size was calculated using Excel software (2010, Microsoft, Redmond, WA, USA) and interpreted based on the following criteria: $<0.20=$ trivial; $0.20-0.59=$ small; $0.60-$ $1.19=$ moderate; $1.20-2.0=$ large $>2.0=$ very large (Hopkins, 2002).

\section{Results}

During concentric and eccentric testing (Table 1), second league athletes produced slightly higher peak torque values in the left leg compared to the right leg $(\mathrm{ES}=0.29, \mathrm{p}=0.001,5.5 \%)$. Second league athletes produced slightly lower values of concentric strength during extension $(E S=0.55, p$ $<0.001,9.3 \%$ and $\mathrm{ES}=0.50, \mathrm{p}=0.002,8.0 \%$ for the 
right and left legs, respectively) and moderately lower values during flexion $(E S=0.67, p<0.001$, $12 \%$ and $\mathrm{ES}=0.76, \mathrm{p}<0.001,13 \%$ for the right and left legs, respectively) actions. Second league athletes also produced slightly lower values of eccentric strength during flexion ( $E S=0.55, \mathrm{p}=$ $0.001,10 \%$ and $E S=0.24, p=0.130,4.5 \%$ for the right and left legs, respectively) and extension (ES $=0.46, \mathrm{p}=0.005,9.0 \%$ and $\mathrm{ES}=0.39, \mathrm{p}=0.014$, $7.3 \%$ for the right and left legs, respectively) actions compared to first league athletes.

\begin{tabular}{|c|c|c|c|c|}
\hline \multicolumn{5}{|c|}{$\begin{array}{l}\text { Table } 1 \\
\text { Concentric and eccentric peak torque between the right and left legs of first } \\
\text { and second league athletes evaluated at an angular velocity of } 60^{-0} \cdot \mathrm{s}^{-1} ; \text { mean } \pm S D .\end{array}$} \\
\hline \multirow{2}{*}{ Variables } & \multicolumn{2}{|c|}{ First League } & \multicolumn{2}{|c|}{ Second League } \\
\hline & Right & Left & Right & Left \\
\hline \multicolumn{5}{|l|}{ Concentric peak torque $(\mathrm{N} \cdot \mathrm{m})$} \\
\hline $\begin{array}{l}\text { Extension } \\
\text { (Q) }\end{array}$ & $258 \pm 49$ & $256 \pm 46$ & $234 \pm 37++$ & $235 \pm 35+$ \\
\hline Flexion $(\mathrm{H})$ & $156 \pm 28$ & $155 \pm 28$ & $138 \pm 27++$ & $135 \pm 24++$ \\
\hline \multicolumn{5}{|l|}{ Eccentric peak torque $(\mathrm{N} \cdot \mathrm{m})$} \\
\hline Flexion (Q) & $304 \pm 61$ & $302 \pm 59$ & $273 \pm 52++$ & $288 \pm 53^{*}$ \\
\hline $\begin{array}{l}\text { Extension } \\
(\mathrm{H})\end{array}$ & $181 \pm 37$ & $176 \pm 34$ & $164 \pm 34 \dagger$ & $163 \pm 31+$ \\
\hline \multicolumn{5}{|c|}{$\begin{array}{l}\text { Abbreviations: } \mathrm{s} \text {, second; } \mathrm{SD} \text {, standard deviation; } \mathrm{N} \cdot \mathrm{m}, \text { Newton-metre; } \mathrm{Q} \text {, } \\
\text { quadriceps; H, hamstrings; } * \text {, significantly different between legs }(p<0.05) ; \mathrm{t}, \\
\text { significantly different between the first and second league }(p<0.05) ;+ \text {, } \\
\text { significantly different between the first and second league }(p<0.001) .\end{array}$} \\
\hline
\end{tabular}

\begin{tabular}{|c|c|c|c|c|}
\hline \multicolumn{5}{|c|}{$\begin{array}{l}\text { Strength ratios between the right and left legs of first } \\
\text { and second league athletes evaluated at an angular velocity of } 60^{\circ} \cdot \mathrm{s}^{-1} ; \text { mean } \pm S D \text {. }\end{array}$} \\
\hline \multirow{2}{*}{ Variables } & \multicolumn{2}{|c|}{ First League } & \multicolumn{2}{|c|}{ Second League } \\
\hline & Right & Left & Right & Left \\
\hline $\begin{array}{l}\text { Conventional Strength Ratio } \\
\left(\mathrm{H}_{\mathrm{con} /} / \mathrm{Q}_{\mathrm{con}}\right)\end{array}$ & $0.62 \pm 0.10$ & $0.61 \pm 0.11$ & $0.59 \pm 0.10$ & $0.58 \pm 0.09+$ \\
\hline $\begin{array}{l}\text { Dynamic Control Ratio } \\
\text { (Hecc/Q }\end{array}$ & $0.71 \pm 0.12$ & $0.70 \pm 0.14$ & $0.71 \pm 0.15$ & $0.70 \pm 0.13$ \\
\hline
\end{tabular}




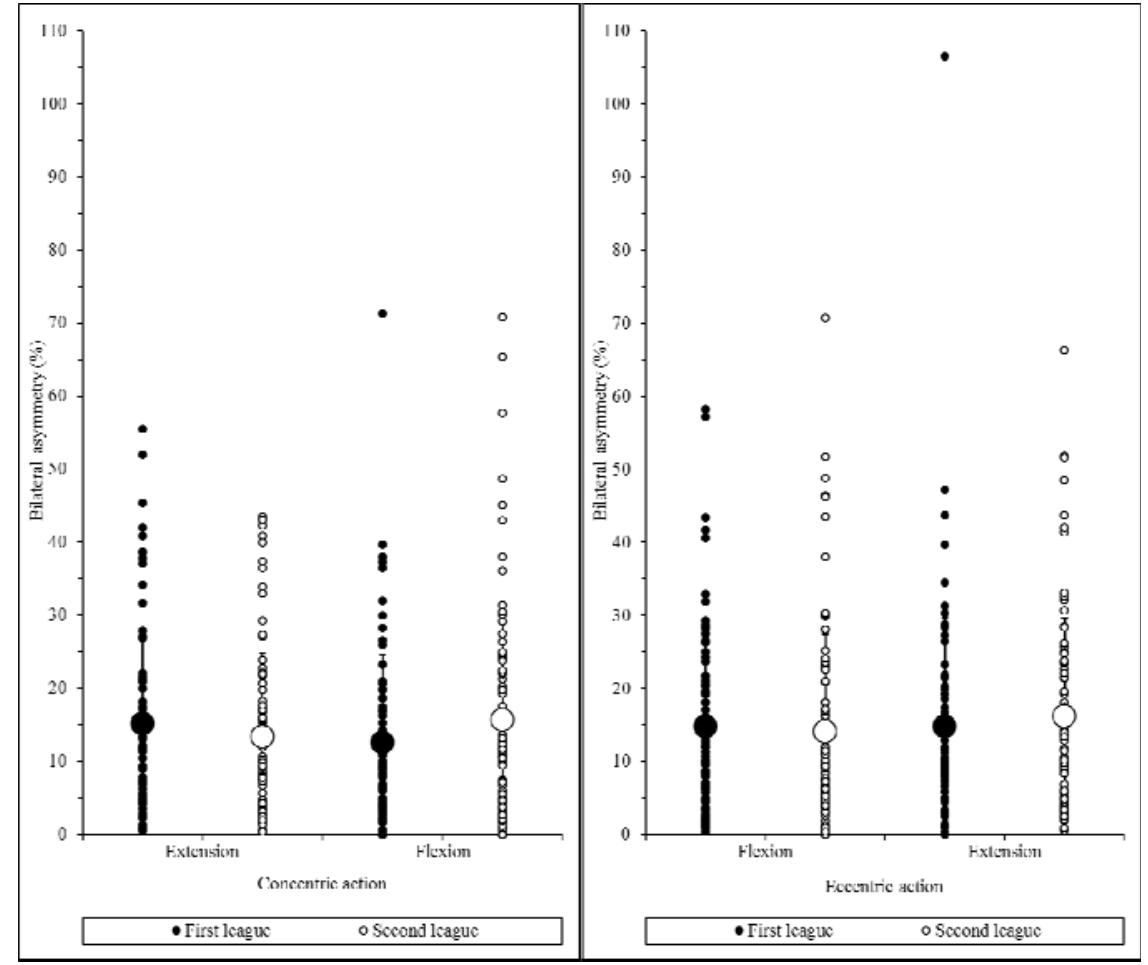

Figure 1

Individual bilateral asymmetry during concentric and eccentric extension and flexion actions at an angular velocity of $60^{\circ} \cdot s-1$ between first and second league athletes; mean $\pm S D$.

Second league athletes produced slightly lower CSRs in the right and left legs ( $E S=0.22, \mathrm{p}=0.174$, $3.5 \%$ and $\mathrm{ES}=0.36, \mathrm{p}=0.023,6.0 \%$, respectively) compared to the first league athletes. No differences were found in DCRs in the either leg between first and second league athletes (Table 2).

No differences were found in bilateral asymmetry during concentric extension and flexion actions between the first and second league athletes (Figure 1).

\section{Discussion}

The primary aim of this research was to compare muscle strength imbalances between professional soccer players of different performance levels (i.e. first and second league). The current study identified several clear and substantial strength deficits between two highlevel professional soccer teams. We found that second league athletes produced substantially lower peak torque values across all concentric and eccentric actions measured compared to the first league athletes. Accompanied by these lower peak torque values, second league athletes also presented lower conventional strength ratios in both legs compared to the first league athletes. No significant differences were found in dynamic control ratios or in bilateral asymmetry among first and second league athletes.

Isokinetic strength has been used in a variety of sports to identify strength deficits. Offseason training is an important phase to detect and identify these strength and motor deficiencies and is usually characterized by high volume and frequency of training to build foundations for sport-specific development in later phases (Gamble, 2010). First league athletes typically perform a longer off-season period compared to second league, which focuses on individual preparation with specific strength training to 
increase muscle mass and muscle strength. This longer training stimulus pared with a higher level of professional match play may result in the increased strength found in the current study.

The CSR values found in first league athletes are in accordance with the accepted normal ratio $(\geq 0.60)$ frequently used as an injury prevention tool (Kannus, 1994). The CSR values found in second league athletes, although very close to normative values, were lower compared to the first league athletes. These results are directly affected by the peak torque values examined, so a lower CSR was expected. As a current debate exists regarding the validity of CSRs, caution should be applied when interpreting these results as CSRs only assess the concentric ability of the muscles in relation to each other and do not reflect the functional movements of sporting activities (e.g. the interaction between eccentric hamstrings and concentric quadriceps seen during kicking) (Coombs and Garbutt, 2002). In line with current research, the DCR was also performed to accurately represent the dynamic relationship between the hamstrings and quadriceps during knee extension. First and second league athletes presented similar ratios of $\sim 0.70$. These values are far from the recommended (Coombs and Garbutt, 2002) value of 1.0 which represents the point of equality, indicating that eccentrically acting hamstrings have the ability to fully brake the action of the concentrically contracting quadriceps. As a consequence of insufficient eccentric capacity of the hamstring muscles to offset the concentric action of the quadriceps during terminal swing, increased injury risk in the lower extremities may result (Croisier et al., 2002).

Second league athletes presented lower bilateral asymmetry in the quadriceps during concentric $(12 \%)$ and eccentric $(4.7 \%)$ actions, but substantially greater bilateral asymmetry in the hamstrings during concentric $(-25 \%)$ and eccentric $(-10 \%)$ actions compared to the first league athletes. The specific dynamic nature of soccer requires the unilateral use of the lower extremities in almost all kicking and cutting skills (Reilly, 1997), which may alter the strength balance between lower extremities or between antagonistic muscle groups (Fousekis et al., 2010). Previous research (Croisier et al., 2002) identified that the strength status of normalized muscle strength corresponded to a less than $5 \%$ deficit through bilateral comparison. The bilateral asymmetry during concentric extension and flexion for first league (ranging between 1-55\% and $0-71 \%$, respectively) and second league athletes (ranging between $0-44 \%$ and $0-71 \%$, respectively) and during eccentric flexion and extension for first league (ranging between $0-58 \%$ and $0-107 \%$, respectively) and second league athletes (ranging between $0-71 \%$ and $1-66 \%$, respectively) may represent an increased risk of injury, particularly in hamstrings (Burkett, 1970). These findings present a major concern to the athlete and the team's personnel. A recent investigation (Carroll et al., 2006) suggested that performing unilateral strength training exercises may be important to increase the efficiency of bilateral movements, promoting contralateral effects and decreasing the risk of injury. Thus, it seems that strength and conditioning coaches should promote unilateral training (e.g. split squats, forward and reverse lunges, single-leg dead lifts, single leg back extensions, etc.) to correct bilateral asymmetry in professional soccer athletes with substantial bilateral differences.

As a result of the substantial strength differences found in the current study, second league athletes would most certainly benefit from adopting pre-season assessments to identify those individuals that may be at risk of injury and then performing individualized training programs (comparable to first league athletes) to reduce asymmetry and increase strength. Following this initial assessment, strength and conditioning personnel would possess the ability to monitor athletes across a training progression and over a season to ensure an appropriate stimulus is applied, with the ultimate goal of reducing the risk of hamstrings injury. An additional feature of this assessment would be the ability to compare an individual athlete to a first league athlete in hopes of progressing them to a higher level of professional soccer (i.e. first league). However, some caution should be applied when interpreting these results, since the differences of a movement pattern between isokinetic assessment and sport specific movements might lead to differences in the identification of bilateral differences. Thus, coaches should constantly monitor the training process in order to address the muscular imbalances applying sport specific 
movements and/or isokinetic training sessions.

\section{Conclusions}

Second league soccer players produced lower isokinetic strength during concentric and eccentric actions and lower CSRs compared to first league athletes. However, no significant bilateral hamstring asymmetry was found. These findings may raise a concern among second league players in terms of injury risk and athletic performance when compared to the next (highest) level of professional soccer that they may be seeking to progress into. However, caution should be applied when interpreting these results. Despite the difficulty of assuming an obvious relationship between the competitive level and risk of injury in Portuguese soccer players, the differences found in conventional strength ratios reinforce the importance of performing off-season and pre-season strength assessments to prescribe and adjust individual strength training programs among professional soccer players. Thus, offseason and pre-season periods should focus on increasing muscle strength and decreasing muscle imbalances in the lower extremities. For instance, unilateral and eccentric strength training may be considered very important strategies to diminish bilateral hamstring asymmetry. Keeping that in mind, professional soccer clubs must encourage technical staff to include fitness coaches specialized in strength and conditioning training to prevent muscular injuries. Strength and conditioning personnel should consider more unilateral strengthening regimes aimed first at unilateral hamstrings strength and then at bilateral asymmetry. The results of the current study further perpetuate the need for pre-season strength assessments, individual programming and the continuation of strength monitoring among second league soccer players.

\section{References}

Aagaard P, Simonsen EB, Trolle M, Bangsbo J, Klausen K. Isokinetic hamstring/quadriceps strength ratio: influence from joint angular velocity, gravity correction and contraction mode. Acta Physiol Scand, 1995; 154(4): 421-427

Askling CM, Tengvar M, Saartok T, Thorstensson A. Acute first-time hamstring strains during slow-speed stretching: clinical, magnetic resonance imaging, and recovery characteristics. Am J Sports Med, 2007; 35(10): 1716-1724

Bangsbo J, Mohr M, Krustrup P. Physical and metabolic demands of training and match-play in the elite football player. J Sports Sci, 2006; 24(7): 665-674

Bardis C, Kalamara E, Loucaides G, Michaelides M, Tsaklis P. Intramachine and intermachine reproducibility of concentric performance: A study of the Con-Trex MJ and the Cybex Norm dynamometers. Isokinet Exerc Sci, 2004; 12(2): 91-97

Brown SR, Brughelli M, Griffiths PC, Cronin JB. Lower-extremity isokinetic strength profiling in professional rugby league and rugby union. Int J Sports Physiol Perform, 2014; 9(2): 358-361

Burkett LN. Causative factors in hamstring strains. Medicine and science in sports, 1970; 2(1): 39-42

Capranica L, Battenti M, Demarie S, Figura F. Reliability of isokinetic knee extension and flexion strength testing in elderly women. J Sport Med Phys Fit, 1998; 38(2): 169-176

Carroll TJ, Herbert RD, Munn J, Lee M, Gandevia SC. Contralateral effects of unilateral strength training: evidence and possible mechanisms. J Appl Physiol (1985), 2006; 101(5): 1514-1522

Carvalho A, Caserotti P, Carvalho C, Abade E, Sampaio J. Reliability of Concentric, Eccentric and Isometric Knee Extension and Flexion when using the REV9000 Isokinetic Dynamometer. Journal of Human Kinetics, 2013; 37: 4753

Cheung RT, Smith AW, Wong del P. H:q ratios and bilateral leg strength in college field and court sports players. J Hum Kinet, 2012; 33: 63-71

Chumanov ES, Heiderscheit BC, Thelen DG. Hamstring musculotendon dynamics during stance and swing phases of high-speed running. Med Sci Sports Exerc, 2011; 43(3): 525-532

Coombs R, Garbutt G. Developments in the use of the hamstring/quadriceps ratio for the assessment of muscle balance. Journal of Sports Science and Medicine, 2002; 1(3): 56-62

Croisier JL. Factors associated with recurrent hamstring injuries. Sports Med, 2004; 34(10): 681-695

Croisier JL, Crielaard JM. Hamstring muscle tear with recurrent complaints: An isokinetic profile. Isokinetics and Exercise Science, 2000; 8: 175-180 
Croisier JL, Forthomme B, Namurois MH, Vanderthommen M, Crielaard JM. Hamstring muscle strain recurrence and strength performance disorders. Am J Sports Med, 2002; 30(2): 199-203

Croisier JL, Ganteaume S, Binet J, Genty M, Ferret JM. Strength imbalances and prevention of hamstring injury in professional soccer players: a prospective study. Am J Sports Med, 2008; 36(8): 1469-1475

Drouin JM, Valovich-mcLeod TC, Shultz SJ, Gansneder BM, Perrin DH. Reliability and validity of the Biodex system 3 pro isokinetic dynamometer velocity, torque and position measurements. Eur J Appl Physiol, 2004; 91(1): $22-29$

Ekstrand J, Hagglund M, Walden M. Epidemiology of Muscle Injuries in Professional Football (Soccer). Am J Sport Med, 2011; 39(6): 1226-1232

Fousekis K, Tsepis E, Vagenas G. Multivariate isokinetic strength asymmetries of the knee and ankle in professional soccer players. J Sports Med Phys Fitness, 2010; 50(4): 465-474

Gamble P. Strength and Conditioning for Team Sports: Sport-Specific Physical Preparation for High Performance. New York: Routledge; 2010

Heiser TM, Weber J, Sullivan G, Clare P, Jacobs RR. Prophylaxis and management of hamstring muscle injuries in intercollegiate football players. Am J Sports Med, 1984; 12(5): 368-370

Hopkins WG. A Scale of magnitudes for effect statistics, 2002 . Available at: http://www.sportsci.org/resource/stats/effectmag.html; accessed in May, 2014

Iga J, George K, Lees A, Reilly T. Reliability of assessing indices of isokinetic leg strength in pubertal soccer players. Pediatr Exerc Sci, 2006; 18(4): 436-445

Impellizzeri FM, Bizzini M, Rampinini E, Cereda F, Maffiuletti NA. Reliability of isokinetic strength imbalance ratios measured using the Cybex NORM dynamometer. Clin Physiol Funct I, 2008; 28(2): 113-119

Kannus P. Isokinetic evaluation of muscular performance: implications for muscle testing and rehabilitation. Int J Sports Med, 1994; 15 Suppl 1: S11-18

Kuruganti U, Seaman K. The bilateral leg strength deficit is present in old, young and adolescent females during isokinetic knee extension and flexion. European journal of applied physiology, 2006; 97(3): 322-326

More RC, Karras BT, Neiman R, Fritschy D, Woo SL, Daniel DM. Hamstrings-an anterior cruciate ligament protagonist. An in vitro study. Am J Sports Med, 1993; 21(2): 231-237

Orchard J. Biomechanics of muscle strain injury. New Zealand Journal of Sports Medicine, 2002; 30: 92-98

Ordway NR, Hand N, Briggs G, Ploutz-Snyder LL. Reliability of knee and ankle strength measures in an older adult population. J Strength Cond Res, 2006; 20(1): 82-87

Orri JC, Darden GF. Technical report: Reliability and validity of the iSAM 9000 isokinetic dynamometer. J Strength Cond Res, 2008; 22(1): 310-317

Reilly T. Energetics of high-intensity exercise (soccer) with particular reference to fatigue. J Sports Sci, 1997; 15(3): 257-263

Ruas CV, Minozzo F, Pinto MD, Brown LE, Pinto RS. Lower-extremity strength ratios of professional soccer players according to field position. J Strength Cond Res, 2015; 29(5): 1220-1226

Sole G, Hamren J, Milosavljevic S, Nicholson H, Sullivan SJ. Test-retest reliability of isokinetic knee extension and flexion. Arch Phys Med Rehab, 2007; 88(5): 626-631

Thelen DG, Chumanov ES, Best TM, Swanson SC, Heiderscheit BC. Simulation of biceps femoris musculotendon mechanics during the swing phase of sprinting. Med Sci Sports Exerc, 2005; 37(11): 1931-1938

\section{Corresponding author:}

\section{Eduardo Abade}

Research Center in Sports Sciences, Health Sciences and Human Development, CIDESD, University Institute of Maia, ISMAI, Portugal.

ISMAI - Instituto Superior da Maia. Av. Carlos Oliveira Campos - Castelo da Maia. 4475-690 Avioso S. Pedro Phone number: +351 229825319

Fax: +351 229825331

E-mail: eduardoabade@gmail.com 